\title{
Treatment of osteolytic solitary painful osseous metastases with radiofrequency ablation or cryoablation: A retrospective study by propensity analysis
}

\author{
LUIGI ZUGARO $^{1 *}$, MARIO DI STASO ${ }^{2 *}$, GIOVANNI LUCA GRAVINA $^{2 *}$, PIERLUIGI BONFILI ${ }^{2}$, \\ LORENZO GREGORI $^{1}$, PIETRO FRANZESE ${ }^{2}$, FRANCESCO MARAMPON ${ }^{2}$, \\ VINCENZO TOMBOLINI $^{3}$, ERNESTO DI CESARE ${ }^{2}$ and CARLO MASCIOCCHI $^{1}$ \\ ${ }^{1}$ Department of Biotechnological and Applied Clinical Sciences, Division of Radiology; \\ ${ }^{2}$ Department of Biotechnological and Applied Clinical Sciences, Division of Radiation Oncology and Radiobiology, \\ San Salvatore Hospital, University of L'Aquila Medical School, L'Aquila 67100; ${ }^{3}$ Department of Radiological, \\ Oncological and Anatomopathological Sciences, 'Sapienza' University, Rome 00185, Italy
}

Received February 5, 2015; Accepted December 10, 2015

DOI: $10.3892 / 01.2016 .4108$

\begin{abstract}
The present study aimed to measure the improvement in pain relief and quality of life in patients with osteolytic solitary painful bone metastasis treated by cryoablation (CA) or radiofrequency ablation (RFA). Fifty patients with solitary osteolytic painful bone metastases were retrospectively studied and selected by propensity analysis. Twenty-five patients underwent $\mathrm{CA}$ and the remaining twenty-five underwent RFA. Pain relief, in terms of complete response (CR), the number of patients requiring analgesia and the changes in self-rated quality of life (QoL) were measured following the two treatments. Thirty-two percent of patients treated by CA experienced a CR at 12 weeks versus $20 \%$ of patients treated by RFA. The rate of CR increased significantly with respect to baseline only in the group treated by CA. In both groups there was a significant change in the partial response with respect to baseline (36\% in the CA group vs. $44 \%$ in the RFA group). The recurrence rate in the CA and RFA groups was $12 \%$ and $8 \%$, respectively. The reduction in narcotic medication requirements with respect to baseline was only significant in the group treated by CA. A significant improvement in self-rated QoL was observed in both groups. The present study seems to suggest that CA only significantly improves the rate of $\mathrm{CR}$ and decreases the requirement of narcotic medications.
\end{abstract}

Correspondence to: Dr Giovanni Luca Gravina, Department of Biotechnological and Applied Clinical Sciences, Division of Radiation Oncology and Radiobiology, San Salvatore Hospital, University of L'Aquila Medical School, via Vetoio 2/A, L'Aquila 67100, Italy

E-mail: giovanniluca.gravina@libero.it

*Contributed equally

Key words: cryoablation, radiofrequency ablation, bone metastases, quality of life, pain control, cancer
Both CA and RFA led to an improvement in the self-rated QoL of patients after the treatments. However, the results of the present study should be considered as preliminary and to serve as a framework around which future trials may be designed.

\section{Introduction}

Metastases are the major cause of cancer-associated death in cancer patients, and are the predominant sites to which metastasis occurs $(1,2,3)$. The primary tumors that most frequently metastasize to the bones include breast, prostate and lung cancer (4-6). Pain is the most common symptom experienced by patients, among all potential complications associated with bone metastasis, and results in a significant deterioration in quality of life (QoL) $(1,4,5,7,8)$. Current treatment objectives are designed to decrease the tumor burden in patients with overt bone metastases, maximize pain control and reduce the incidence of skeletal-related events (5). Pain management/analgesia, surgery, radiation therapy (RT), systemic therapy, or a combination of these techniques are current strategies for the management of skeletal metastasis (9). However, the long-term results of these treatments require improvement $(10,11)$.

Thermoablation is a category of non-surgical approaches used to treat otherwise unresectable tumors, and the techniques it includes utilize a variety of power sources. Furthermore, an array of delivery methods for ablation exist, including laser ablation, radiofrequency ablation (RFA), cryoablation (CA), microwave ablation and high intensity focused ultrasound (HIFU) (12-14). These techniques have proven to be useful therapeutic options for bone tumor management, in addition to the treatment of metastatic liver and malignant primary tumors for which they were originally developed (15-18). Recent studies have demonstrated that the association of RT with RFA is well tolerated, and has a satisfactory profile of adverse side effects $(19,20)$. The aim of the present study was to investigate whether RFA and CA improve the clinical management of osteolytic solitary painful bone metastases, 
selecting the study population retrospectively by propensity analysis.

\section{Materials and methods}

Patient selection. In the present study, patients $>18$ years, with painful solitary osteolytic bone metastases, confirmed by radiological and histological investigations were retrospectively selected from a pool of subjects who underwent CA or RFA between September 2011 and September 2014. Magnetic resonance imaging (MRI) examination, computed tomography (CT), and nuclear isotope (technetium 99) bone scans were performed within the 4 weeks prior to the procedures. Eligibility criteria included: (i) A pain score of $\geq 5$ on the validated visual analog scale (VAS) over the previous 24 hours (or a score of $<5$ with the use of narcotic medications); (ii) pain localized to the site of the bone metastases; (iii) life expectancy $>3$ months, and (iv), a Karnofsky performance status (KPS) score $>70$. Exclusion criteria were: (i) The area of pain had been previously treated with RT, palliative surgery or interventional radiological approaches; (ii) radiographic evidence of spinal cord or cauda-equina compression; (iii) lesions positioned within $0.5 \mathrm{~cm}$ of a critical site, such as the spinal cord, brain, aorta, inferior vena cava, bowel, or bladder; (iv) abnormal fracture of the treatment site. Written informed consent was obtained from all patients. The present retrospective study was approved by the San Salvatore Hospital institutional Ethical Committee.

Cryoablation. Percutaneous CA was carried out under conscious sedation with an argon-based cryotherapy system (SeedNet ${ }^{\circledR}$ Gold Cryoablation System, Galil Medical Ltd., Yokneam, Israel). Following sterile preparation, one or more cryoprobes (IceRod ${ }^{\circledR}$, Galil Medical Ltd.) were introduced into the target lesion with $\mathrm{CT}$ guidance by experienced radiologists. The cryoprobes were introduced in a parallel arrangement $\sim 2 \mathrm{~cm}$ apart. For larger lesions, a cluster of cryoprobes was placed within $1 \mathrm{~cm}$ of the tumor margin to provide adequate coverage of the outer border of the target lesion. Cryoprobe positioning was confirmed by CT imaging. Rapid freezing of the target lesion $\left(-100{ }^{\circ} \mathrm{C}\right.$ within a few seconds) was performed, and two $15 \mathrm{~min}$ freezes separated by a $10 \mathrm{~min}$ thaw were used at each cryoprobe position.

Radiofrequency ablation. Percutaneous RFA was performed under conscious sedation. Following sterile preparation, a LeVeen $^{\mathrm{TM}}$ needle electrode (Boston Scientific Corp., Natick, MA, USA) was introduced under CT guidance into the metastases. After unfolding the electrode tines into the metastases, the needle was connected to a radiofrequency generator ( $\mathrm{RF}$ $3000^{\circledR}$; Boston Scientific Corp.). The procedure was conducted according to the protocols supplied by the equipment manufacturers. Briefly, the developed energy was increased $10 \mathrm{~W}$ every 3 min up to $90 \mathrm{~W}$, until tissue impedance increased and further current flow was prevented (roll-off). A target intratumoural temperature higher than $60{ }^{\circ} \mathrm{C}$ was considered as an indicator of adequate thermocoagulation. A single ablation was performed for lesions measuring $<3 \mathrm{~cm}$ in the longest diameter. For larger lesions $(3-7 \mathrm{~cm})$ a cluster RFA electrode technique was used ( 3 needles spaced $5 \mathrm{~mm}$ apart). At the end of each CA or RFA, a CT was performed to ensure that the extent of ablation was confined to the target tissue and that there was no substantial damage in the tissue surrounding the target. After all combined procedures, patients were observed for $2 \mathrm{~h}$ in the recovery room and were then admitted to the hospital for a minimum of $24 \mathrm{~h}$.

Patient assessment. A full physical examination was performed and data on direct and indirect changes in pain levels were assessed by VAS and a medication level questionnaire. QoL was assessed with a single question from the McGill Quality of Life Questionnaire (MQOL) (21). The strongest evidence of validity comes from comparison with the single-item quality of life measure (21).

Complications of the procedures. Complications related to ablative approaches were rated as major or minor according to the guidelines of the Society of Interventional Radiology (22).

Study endpoints and response criteria. The primary endpoints were to assess the changes from baseline (pre-CA or RFA) to week 12 in the rate of (1) complete (CR) and (2) partial (PR) responses (23). The secondary endpoints were to assess the changes from baseline (pre-CA or RFA) to week 12 in the rate (1) of subjects requiring opioid analgesic use and (2) in self-experienced QoL.

Statistical analysis. The data analyzed in the present study were derived from a population-based retrospective study. In order to reduce treatment selection bias and realistically determine the treatment effects, a case control-matched propensity analysis was performed. Multivariate logistic regression was used to calculate the predicted probability of the dependent variables, as well as the propensity score for all observations in the dataset. The dependent variables included in the multivariate analysis were age, KPS, primary tumor number, metastasis location, VAS scale, and the QoL prior to the procedures. A 1:1 matched analysis was performed, where one subject treated by CA was matched to one subject treated by RFA. Continuous variables not normally distributed (Shapiro-Wilk test) were presented as medians and 95\% confidence intervals (95\% CI). The Mann-Whitney U test was used to evaluate the difference between two groups and the Kruskal-Wallis test was used to evaluate the difference between more than two tests. If the Kruskal-Wallis test was statistically significant, a pairwise comparison of subgroups was performed according to Conover. Dichotomous variables were summarized by absolute and/or relative frequencies. The Chi-squared test or Fisher's exact test was used to evaluate the difference between two groups. For multiple comparisons, the alpha value threshold was adjusted by using Bonferroni correction. For matched pairwise multiple comparisons, dichotomous variables were compared with Cochran's Q test. For matched pair-wise comparisons McNemar's test was used. All tests were two-sided except where specified, and were determined by Monte Carlo significance. An alpha value threshold of 0.05 was used. All statistical analyses were performed using the SPSS ${ }^{\circledR}$ statistical analysis software package, version 10.0 (SPSS, Inc., Chicago, IL, USA). 
Table I. Clinical characteristics according to propensity score

\begin{tabular}{|c|c|c|c|}
\hline Characteristics & RFA $(n=25)$ & $\mathrm{CA}(\mathrm{n}=25)$ & P-value \\
\hline Age, years (range) & $66(59-70)$ & $67.5(64.4$ to 70.6$)$ & $0.678^{\mathrm{a}}$ \\
\hline VAS scale (range) & $7(5-7)$ & $7.5(5-7.6)$ & $0.822^{\mathrm{a}}$ \\
\hline \multicolumn{4}{|l|}{ Sex, no. (\%) } \\
\hline Male & $11(44)$ & $12(48)$ & \multirow[t]{2}{*}{$1.0^{\mathrm{c}}$} \\
\hline Female & $14(56)$ & $13(52)$ & \\
\hline \multicolumn{4}{|l|}{ KPS, no. (\%) } \\
\hline $91-100$ & $10(40)$ & $11(44)$ & \multirow[t]{2}{*}{$1.0^{\mathrm{b}}$} \\
\hline $70-89$ & $15(60)$ & $14(56)$ & \\
\hline Tumor size, $\mathrm{cm}$ & $4(4-5)$ & $4(3.4-6)$ & $0.456^{\mathrm{a}}$ \\
\hline \multicolumn{4}{|c|}{ Primary tumors, no. $(\%)$} \\
\hline Lung cancer & $7(28)$ & $7(28)$ & \multirow{5}{*}{$0.79^{\mathrm{b}}$} \\
\hline Prostate cancer & $2(8)$ & $3(12)$ & \\
\hline Renal cancer & $3(12)$ & $4(16)$ & \\
\hline Colorectal cancer & $4(16)$ & $2(8)$ & \\
\hline Breast cancer & $14(56)$ & $9(36)$ & \\
\hline \multicolumn{4}{|c|}{ Metastasis location, no. (\%) } \\
\hline Pelvis & $10(40)$ & $8(32)$ & \multirow{6}{*}{$0.99^{\mathrm{b}}$} \\
\hline Sacrum & $6(24)$ & $7(28)$ & \\
\hline Rib & $2(8)$ & $2(8)$ & \\
\hline Vertebrae & $3(12)$ & $4(16)$ & \\
\hline Humerus & $2(8)$ & $2(8)$ & \\
\hline Femur & $2(8)$ & $2(8)$ & \\
\hline \multicolumn{4}{|c|}{ Medical systemic treatments, no. $(\%)^{\mathrm{d}}$} \\
\hline Bisphosphonates & $5(20)$ & $8(32)$ & $0.52^{\mathrm{c}}$ \\
\hline Narcotic analgesics & $25(100)$ & $25(100)$ & $1.0^{\mathrm{b}}$ \\
\hline Hormonal therapy & $9(36)$ & $8(32)$ & $1.0^{\mathrm{c}}$ \\
\hline Chemotherapy & $18(72)$ & $15(60)$ & $0.55^{\mathrm{b}}$ \\
\hline Immunotherapy & $2(8)$ & $4(12)$ & $0.67^{\mathrm{c}}$ \\
\hline
\end{tabular}

In post hoc pairwise comparisons of subgroups, the alpha error was set at 0.016 according to Bonferroni correction. ${ }^{a}$ Kruskal-Wallis test; ${ }^{\mathrm{b}}$ Chi-Squared test; ' $\mathrm{C}$ Fisher's Exact test; ${ }^{\mathrm{d}}$ the sum of percentage in each group is $>100 \%$, since patients may undergo more than one systemic treatment. KPS, Karnofsky performance status; Medians and CI95\%; RFA, Radiofrequency Ablation; CA, Cryoablation.

\section{Results}

Table I lists the clinical and demographic characteristics of treated patients stratified according to propensity score and treatment received. At 12 weeks following treatment, all patients were analyzed, as the selected patients were long-term survivors of a solitary bone lesion. The VAS scale before treatments was 7 (95\% CI, 5-7) in the group treated by RFA and 7.5 (95\% CI, 5-67.6) in the group treated by CA. The mean tumor size was $4 \mathrm{~cm}$ in both groups. Primary tumor lesions were located in lung, prostate, kidney, colorectum and breast, and the metastatic sites were pelvis, sacrum, rib, vertebrae, humerus and femur (Table I). Eight out of 25 subjects treated by CA $(32 \%)$ experienced a CR at 12 weeks vs. 5 out of 25 patients treated by RFA (20\%) (Fig. 1). Interestingly, when within analysis was performed by the Cochran's Q test, the rate of CR in the CA group significantly increased with respect to the baseline (Fig. 1C). By contrast, this parameter did not significantly increase with respect to baseline in the RFA group (Fig. 1B). Nine out of 25 subjects treated by CA (36\%) experienced a PR at 12 weeks vs. 11 out of 25 of patients treated by RFA (44\%) (Fig. 1). In both groups there was a significant change in the PR with respect to baseline (Fig. $1 \mathrm{C}$ and D). The number of subjects with stable or progressive pain was 8 out $25(32 \%)$ in the CA group and 9 out of $25(36 \%)$ in the RFA group. Three out of $8(37.5 \%)$ patients in the RFA group and 2 out of $9(22 \%)$ patients in the CA group with stable or progressive pain at 12 weeks from treatments, experienced a 3-point fall in pain within the first 2 weeks following the procedure. These patients had recurrent pain of intensity equal to or greater than the worst pain experienced prior to CA or RFA treatments between the 6th and 10th of follow-up, with no difference in the temporal trend between the two groups. All patients received oral narcotic analgesia over the month 


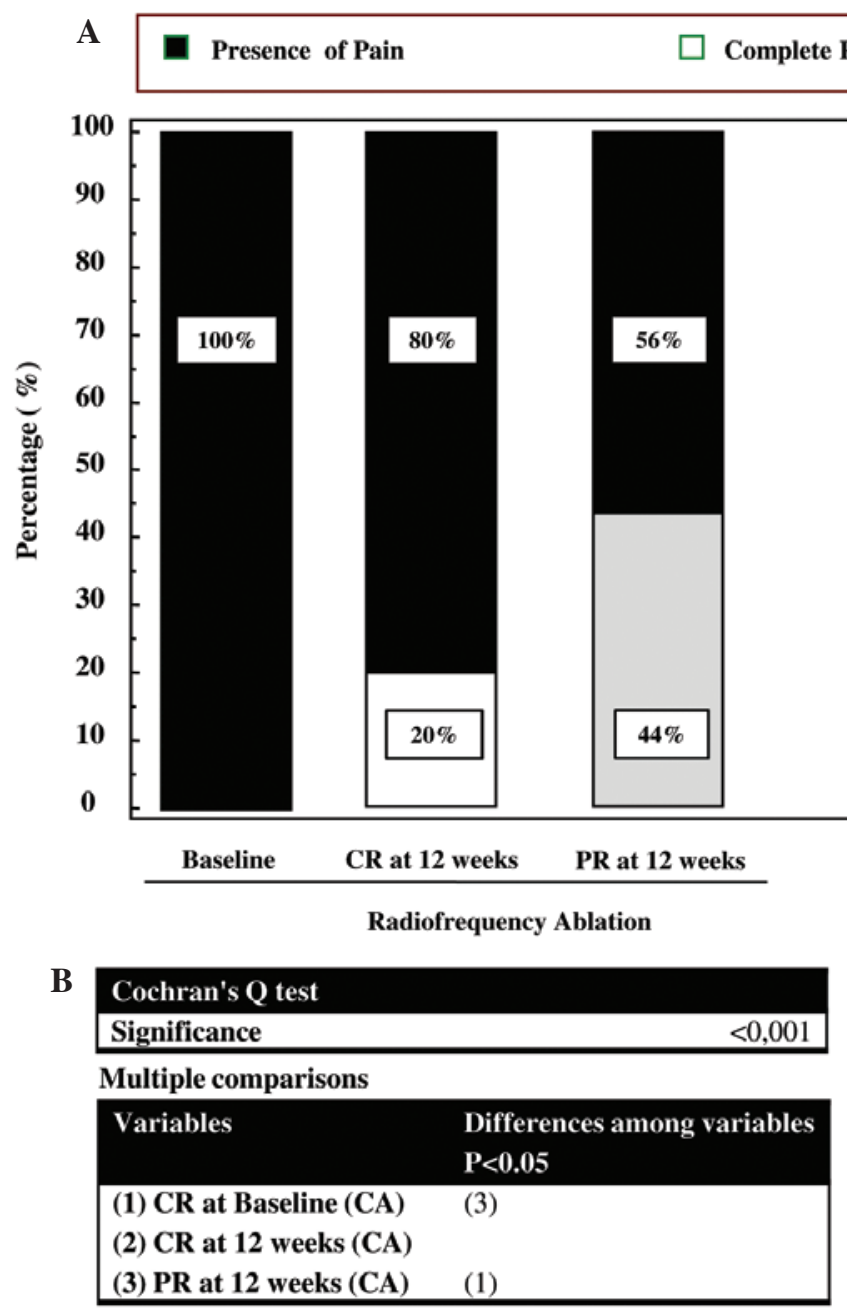

Minimum required difference $(\%): 25,9317$ $\square$ Partial Response (PR)

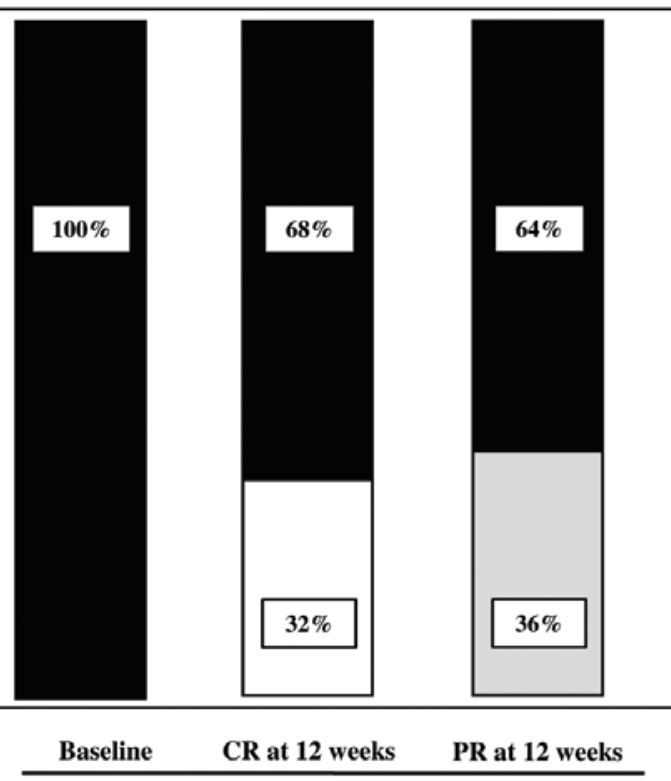

Cryoablation

\begin{tabular}{ll|} 
C & Cochran's Q test \\
\hline Significance & $<0,001$ \\
\hline Multiple comparisons & \\
\hline Varaibles & Differences among variables \\
& $\mathbf{P}<0.05$ \\
\hline \begin{tabular}{ll} 
(1) CR at Baseline (CA) & $(2)(3)$ \\
(2) CR at 12 weeks (CA) & $(1)$ \\
(3) PR at 12 weeks (CA) & (1) \\
\hline
\end{tabular}
\end{tabular}

Minimum required difference $(\%): \mathbf{2 3 , 4 5 6 1}$

Figure 1. (A) Percentage of subjects experiencing Complete Response (CR) and Partial Response (PR) after CA and RFA treatments. Within groups assessment measured at baseline and 12 weeks after treatments. Pairwise multiple comparisons by Cochran's Q test for (B) Radiofrequency ablation and (C) cryoablation. CA, cryoablationl RFA, radiofrequency ablation.

prior to treatment with CA or RFA (Table I). At 12 weeks, $20 \%$ of patients $(5 / 25)$ in the RFA group and $36 \%$ of patients $(9 / 25)$ in the CA group did not require narcotic medication (Fig. 2). For data on the complete and partial response, the $\mathrm{McNemar}$ test indicated that the reduction in narcotic medication requirements was significantly reduced in the CA group $(\mathrm{P}=0.0039)$, whereas in the RFA group, there was a trend toward a lower rate of narcotic used that did not reach statistical significance $(\mathrm{P}=0.062)$. The higher rate of $\mathrm{CR}$ and PR observed in patients treated with CA or RFA correlated with an improved self-rated QoL (Fig. 3). At 12 weeks, patients treated by CA or RFA reported a significant improvement in self-rated QoL. In the group treated by CA, the MQOL score increased from 3.9 [interquartile range (IRQ) 3-4] to 6.1 (IRQ 5.2-8) whereas in the group treated by RFA the score increased from 3.6 (IRQ 3.1-4.2) to 5.6 (IRQ 4.3-7) (Fig. 3). Overall, patients tolerated the CA and RFA treatment. Two transient nerve injuries occurred, one in the RFA group and one in the CA group, neither of which prolonged the length of hospitalization. These injuries appeared 6 and 11 days after the CA and RFA treatments, respectively, with improvement within 10 days following administration of a systemic steroid.

\section{Discussion}

Interventional radiologists have developed a number of ablative techniques for the management of a range of different clinical presentations, including bone metastatic disease, with outstanding results in terms of their efficacy. A relatively limited number of studies have investigated the RFA in the management of metastatic bone lesions. In a previous study, RFA produced a significant reduction in pain, and a significant improvement in the relief felt and in mood, when assessed at one and three months following treatment (16). Another feasibility study confirmed the analgesic efficacy of RFA in absence of serious complication (15). A multicentre trial conducted on 43 patients with painful, refractory, pelvic or sacral lesions evaluated RFA (13). At 4, 12 and 24 weeks following RFA, $95 \%$ of treated patients reported pain relief (a fall of at least two points in the most intense pain) with a reduction in the provision of analgesics observed at 8 and 12 weeks. Complications included cutaneous burn, transitory faecal and urinary incontinence following treatment of a sacral location, and an acetabular fracture. CA is a common ablative technique that creates an 'ice-ball' deep in bone by using miniaturized 


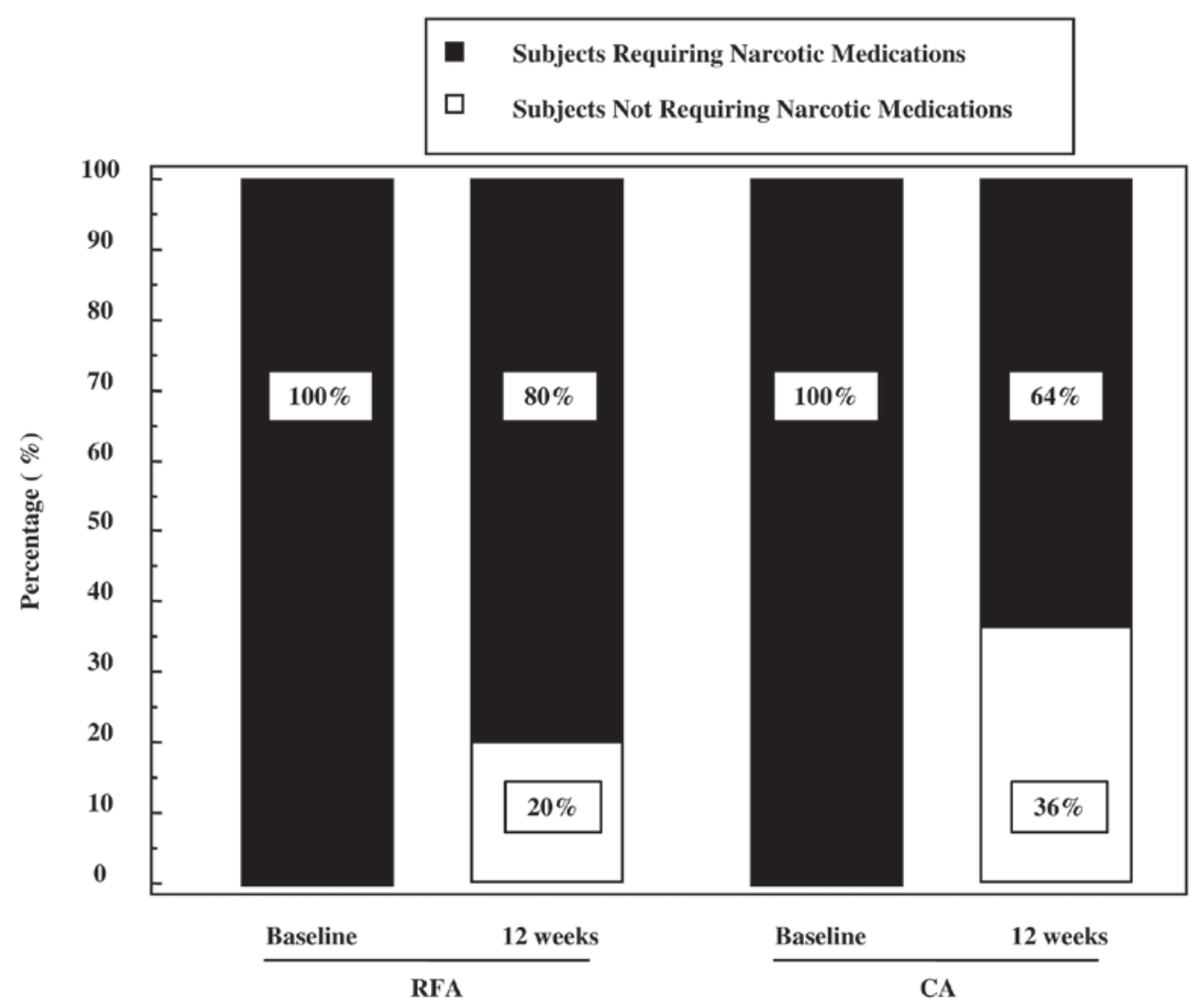

Figure 2. Percentage of patients requiring narcotic medications following CA and RFA treatments. Within groups, assessment was measured at baseline and 12 weeks after treatments by the McNemar test. CA, cryoablationl RFA, radiofrequency ablation.

Box-and-whisker Medians (error bars: $95 \%$ CI for median)

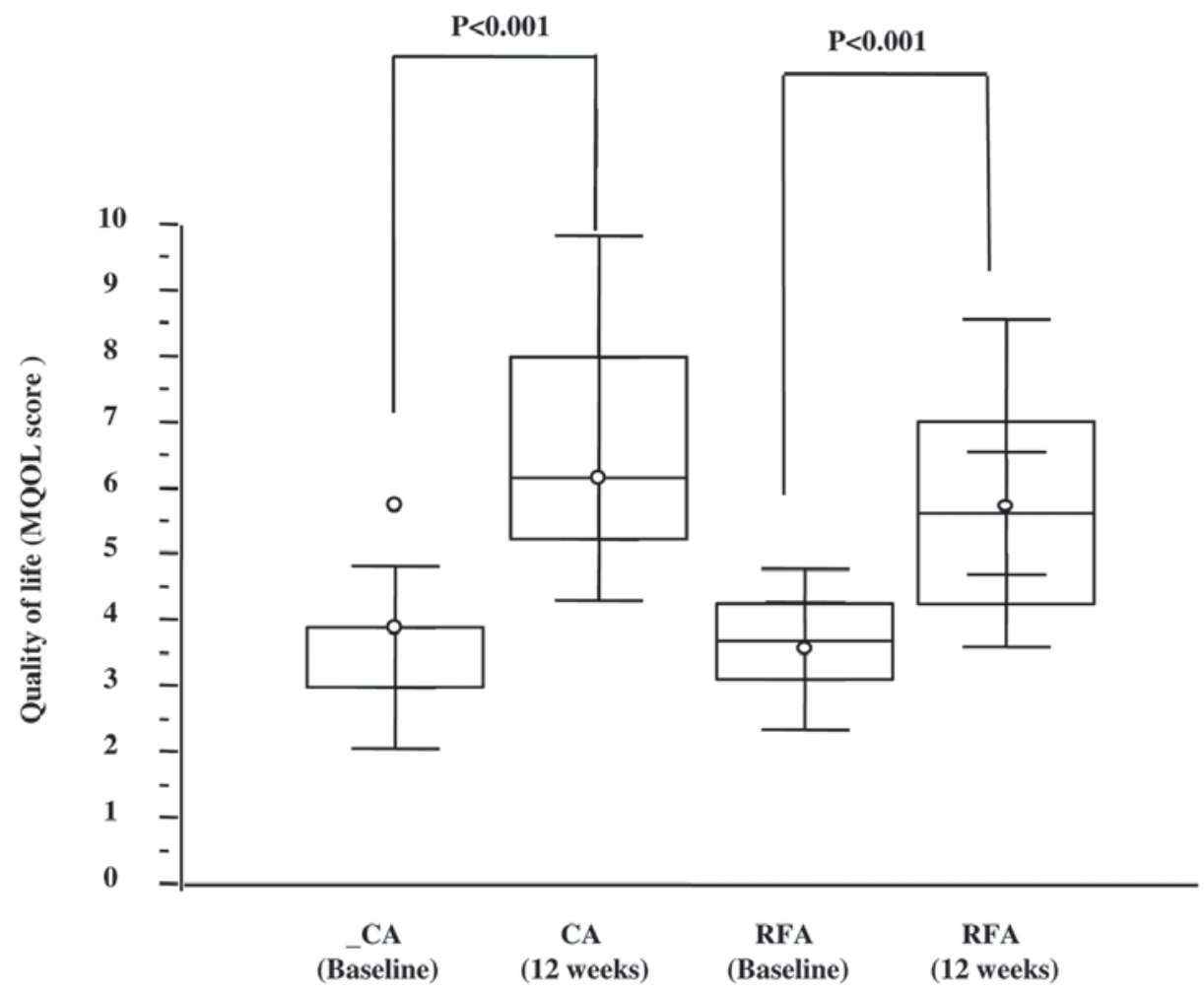

Figure 3. Quality of life score measured by a single question from the MQOL. Within groups assessment of self rated QoL measured at baseline and 12 weeks after treatments. MQOL, McGill quality of life questionnaire; QoL, quality of life, CA, cryoablation; RFA, radiofrequency ablation. 
argon-gas devices. This method is associated with reduced periand post-procedural pain. Recently Callstrom et al $(24,25)$ performed clinical trials in which percutaneous CA was used for the palliation of painful metastatic lesions, and the results demonstrated that CA was safe and effective at reducing the pain associated with metastatic lesions. To date, the precise mechanisms through which CA followed by RT can relieve symptomatic pain remains to be elucidated fully. Pain experienced by patients suffering from bone metastases may have several pathophysiological mechanisms. These include: (i) Microfractures by direct metastatic bone invasion; (ii) periosteum distortion resulting from increased pressure on the endosteum; (iii) nerve-root compression or muscle spasm; and (iv) release of chemical mediators involved in the conduction of nociceptive signals towards the central nervous system $(2,3)$. Reduced cancer cell burden within bone tissue is associated with endosteal pressure reduction. The release and modulation of pain-related chemical mediators with combination treatment may significantly affect the perception of pain intensity.

The current study performed a comparative analysis of the effects that CA or RFA (two well-known interventional ablative approaches) have in relieving cancer pain related to bone metastasis. To the best of our knowledge, this is the first report that analyzes the clinical performances of both RFA and CA in patients with bone metastatic lesions. Of note, the response criteria were defined according to the endpoint criteria of the International Bone Metastases Working Party guidelines on palliative radiotherapy endpoints for future clinical trials. Thus, the results of the present study are comparable with the literature that used the same criteria. Furthermore, the response criteria included changes in narcotic medication use and self-rated QoL data. Our retrospective study design was based on estimates of propensity scores, which can facilitate the generation of unbiased treatment effect estimates. This powerful statistical approach was coupled with the use of a pre- and post-test experimental design. This experimental design is an attractive model for the assessment of clinical efficacy before and following a treatment, as the comparisons were made 'within subjects' rather than 'between subjects'. An important consequence of this experimental strategy is that the study power is greatly improved compared to other research designs.

The present study demonstrated that CA significantly improved both CR and PR with respect to baseline at 12 weeks following ablation. By contrast, only PR significantly improved with respect to baseline following RFA treatment. These data were in line with the improvement of self-rated QoL observed in patients treated by CA or by RFA. When self-rated QoL was assessed from baseline to 12 weeks, the patients treated by the two ablative techniques experienced improved QoL, and this difference was statistically significant. These data are also paralleled with the results demonstrating that $20 \%$ of subjects treated by RFA and $32 \%$ of patients treated by CA reported no opioid analgesic use at 12 weeks. Interestingly, in terms of medical adjunctive treatment, all subjects took narcotic medications prior to treatment. The data concerning the percentage of patients who did not use analgesic medication are in agreement with a study by Callstrom et al (24), who reported that 4 of 19 studied patients were not administered opioid analgesic following CA. Notably, and in agreement with literature, the rate of complications was $4 \%$ following both treatments.
The present study has several limitations, the major ones being the non-randomized study design and limited sample size. Large, randomized controlled clinical trials have provided strong evidence for the efficacy of therapeutic procedures or treatments. Any bias in these studies has been controlled by using a strategy based on propensity score analysis, which can generate groups of patients, randomized post-hoc for important clinical characteristics. The results of the present study have fewer methodological biases from using comparative analysis by propensity-matched pairs, rather than other common statistical methods.

In conclusion, the results of the present study suggest for the first time that CA, in contrast to RFA, significantly improves the rate of $\mathrm{CR}$ and decreases the requirement of narcotic medications. Both CA and RFA improve PR and the self-rated QoL of patients following the treatments. However, our data should be considered as preliminary and to serve as a framework around which to design future trials.

\section{References}

1. Suva LJ, Washam C, Nicholas RW and Griffin RJ: Bone metastasis: Mechanisms and therapeutic opportunities. Nat Rev Endocrinol 7: 208-218, 2011.

2. Roodman GD: Mechanisms of bone metastasis. N Engl J Med 350: 1655-1664, 2004.

3. Suva LJ, Griffin RJ and Makhoul I: Mechanisms of bone metastases of breast cancer. Endocr Relat Cancer 16: 703-713, 2009.

4. Coleman RE: Clinical features of metastatic bone disease and risk of skeletal morbidity. Clin Cancer Res 12: 6243s-6249s, 2006.

5. Coleman RE: Metastatic bone disease: Clinical features, pathophysiology and treatment strategies. Cancer Treat Rev 27: 165-176, 2001.

6. Harvey HA and Cream LV: Biology of bone metastases: Causes and consequences: Clin Breast Cancer 7 (Suppl 1): S7-S13, 2007.

7. Smith HS: Painful osseous metastases. Pain Physician 14: E373-E403, 2011.

8. Tharmalingam S, Chow E, Harris K, Hird A and Sinclair E: Quality of life measurement in bone metastases: A literature review. J Pain Res 1: 49-58, 2008.

9. Yu HH, Tsai YY and Hoffe SE: Overview of diagnosis and management of metastatic disease to bone. Cancer Control 19: 84-91, 2012.

10. Lutz S, Berk L, Chang E, Chow E, Hahn C, Hoskin P, Howell D, Konski A, Kachnic L, Lo S, et al: American Society for Radiation Oncology (ASTRO). Palliative radiotherapy for bone metastases: An ASTRO evidence-based guideline. Int J Radiat Oncol Biol Phys 79: 965-76, 2011.

11. Andrade RS, Proctor JW, Slack R, Marlowe U, Ashby KR and Schenken LL: A simple and effective daily pain management method for patients receiving radiation therapy for painful bone metastases. Int J Radiat Oncol Biol Phys 78: 855-859, 2010.

12. Widmann G, Bodner G, Bale R: Tumour ablation: Technical aspects. Cancer Imaging 9: 63-67, 2009.

13. Goetz MP, Callstrom MR, Charboneau JW, Farrell MA, Maus TP, Welch TJ, Wong GY, Sloan JA, Novotny PJ, Petersen IA, et al: Percutaneous image-guided radiofrequency ablation of painful metastases involving bone: A multicenter study. J Clin Oncol 22: 300-306, 2004.

14. Silverman SG, Tuncali K, Adams DF, vanSonnenberg E, Zou KH, Kacher DF, Morrison PR and Jolesz FA: MR imaging-guided percutaneous cryotherapy of liver tumors: Initial experience. Radiology 217: 657-664, 2000.

15. Callstrom MR, Charboneau JW, Goetz MP, Rubin J, Wong GY, Sloan JA, Novotny PJ, Lewis BD, Welch TJ, Farrell MA, et al: Painful metastases involving bone: Feasibility of percutaneous CT- and US-guided radio-frequency ablation. Radiology 224: 87-97, 2002.

16. Dupuy DE, Liu D, Hartfeil D, Hanna L, Blume JD, Ahrar K, Lopez R, Safran H and DiPetrillo T: Percutaneous radiofrequency ablation of painful osseous metastases: A multicenter American College of Radiology Imaging Network trial. Cancer 116: 989-997, 2010. 
17. Rosenthal D and Callstrom MR: Critical review and state of the art in interventional oncology: Benign and metastatic disease involving bone. Radiology 262: 765-80, 2012.

18. Grieco CA, Simon CJ, Mayo-Smith WW, Dipetrillo TA, Ready NE and Dupuy DE. Image-guided percutaneous thermal ablation for the palliative treatment of chest wall masses. Am J Clin Oncol 30: 361-367, 2007.

19. Di Staso M, Zugaro L, Gravina GL, Bonfili P, Marampon F, Di Nicola L, Conchiglia A, Ventura L, Franzese P, Gallucci M, et al: A feasibility study of percutaneous Radiofrequency Ablation followed by Radiotherapy in the management of painful osteolytic bone metastases. Eur Radiol 21: 2004-2010, 2011.

20. Di Staso M, Zugaro L, Gravina GL, Bonfili P, Marampon F, Di Nicola L, Conchiglia A, Franzese P, Gallucci M, Masciocchi C, et al: Can radiotherapy be combined with radiofrequency ablation in the management of symptomatic osteolytic skeletal metastasis? Clin Oncol (R Coll Radiol) 23: 65-66, 2011.

21. Cohen SR, Mount BM, Strobel MG and Bui F: The McGill Quality of Life Questionnaire: A measure of quality of life appropriate for people with advanced disease. A preliminary study of validity and acceptability. Palliat Med 9: 207-219, 1995.
22. Sacks D, McClenny TE, Cardella JF and Lewis CA: Society of Interventional Radiology clinical practice guidelines. J Vasc Interv Radiol 14: S199-S202, 2003.

23. Chow E, Wu JS, Hoskin P, Coia LR, Bentzen SM and Blitzer PH: International consensus on palliative radiotherapy endpoints for future clinical trials in bone metastases. Radiother Oncol 64:275-280, 2002.

24. Callstrom MR, Dupuy DE, Solomon SB, Beres RA, Littrup PJ, Davis KW, Paz-Fumagalli R, Hoffman C, Atwell TD, Charboneau JW, et al: Percutaneous image-guided cryoablation of painful metastases involving bone: Multicenter trial. Cancer 119: 1033-1041, 2013

25. Callstrom MR, Atwell TD, Charboneau JW, Farrell MA, Goetz MP, Rubin J, Sloan JA, Novotny PJ, Welch TJ, Maus TP, et al: Painful metastases involving bone: Percutaneous image-guided cryoablation--prospective trial interim analysis. Radiology 241: 572-580, 2006. 